\title{
Configurational forces for growth and shape regulations in morphogenesis
}

\author{
P. CIARLETTA ${ }^{1}$, D. AMBROSI ${ }^{2}$, and G.A. MAUGIN ${ }^{1 *}$ \\ ${ }^{1}$ Université Pierre et Marie Curie - Paris 6, Institut Jean le Rond d'Alembert, UMR CNRS 7190, \\ 4 place Jussieu, case 162, 75005 Paris, France \\ ${ }^{2}$ MOX Laboratory, Dipartimento di Matematica, Politecnico di Milano, piazza Leonardo da Vinci 32, 20133 Milan, Italy
}

\begin{abstract}
Morphogenetic theories investigate the mechanisms of creation and regulation of definite biological forms in living organisms. The incredible diversity of shapes and sizes is generated through a barely unknown coordination of biochemical processes occurring at molecular levels. Such a crosstalk not only defines the rules of a robust scheme of matter differentiation, but it also has the capacity to adapt with respect to some variations of the environmental conditions. In this work, we propose a continuum model of growth and mass transport for biological materials during morphogenetic processes. Using the theory of configurational forces, we define the thermomechanical bases for understanding how both the mechanical and the biochemical states can orchestrate growth. The model is successfully applied to describe the morphogen-driven growth control in the imaginal wing disc of Drosophila melanogaster.
\end{abstract}

Key words: growth regulation, remodeling, mechanobiology, embryogenesis, configurational forces.

\section{Introduction}

The first mechanistic treatment of the interrelations between growth and form is due to D'Arcy Thompson, who sought to explain the generation of organic forms from a structural optimization principle under given physical forces [1]. This approach practically identifies form as Goethe's concept of 'Gestalt', being described like a mathematical mapping of the same organic structure. A radical conceptual change later arose thanks to the combined development of genetics and embryology. In 1938, Waddington used the term 'evocator' to indicate a biochemical substance enabling certain potential abilities in the embryonic tissue [2]. This concept was implicitly employed by Turing for the definition of 'morphogens', intended as diffusible secreted substances acting like evocators of shape [3]. In Turing's idea, morphogens behave like activators and inhibitors in chemical reactions, directing the formation of complex patterns from homogeneous states through the coupling between their reaction-diffusion mechanisms. Wolpert later simplified this concept of reacting substances, introducing the seminal idea that cells acquire positional information by reading the local signalling level of diffusive morphogens [4]. In the so-called 'French flag' model, he proposed that target cells might use this information as a paradigm to give rise to spatial patterns, activating different transcription genes depending on given concentration thresholds of the morphogens. This apparently simplistic model was later confirmed by experimental observations on the early Drosophila embryo, where the concentration gradient of the protein $\mathrm{Bi}$ coid (a transcription factor) drives an antero-posterior differentiation in three separated domains, scaling with embryo size like the colours in a flag. Although successful and long time accepted as a universal mechanism, this patterning model is based on two controversial issues, as one can question if morphogens can actually freely diffuse, and if their concentration field is sufficiently stable. Wolpert himself later argued that passive diffusion might not be a reliable mechanism inside cells [5]. Morphogenetic movements are rather determined by other transport mechanisms, such as planar transcytosis (via endocytosis and re-secretion), cytonemes (using actin filaments bridging) or through heparan sulphate proteoglycans (enhancing the spreading at the cell surfaces) [6]. Moreover, graded morphogens generally travel through individual cells with different sizes and mechanical/chemical properties, being subjected to fluctuations on short length-scales, while driving precise positional information. Without discussing in further details such limiting aspects, it is now generally accepted that more complicated morphogenetic models are needed in the wide scenario of embryogenesis. In fact, it has been also pointed out that morphogens have a much broader functionality, behaving as both patterning agents and growth factors [7]. Grafting experiments on amphibians have shown that regeneration, i.e. intercalary growth, occurs between cellular boundaries that are not normally in contact. Furthermore, particular tissue components, called 'organizers', have the ability to coordinate defined structural changes in neighbouring cells, intrinsically carrying information about the final size. Unlike their function in pattern formation, the role of morphogens in the regulation of growth, shape and size is largely unknown. On one hand, their local concentration can trigger an increase of mass resulting from a random cellular proliferation. Preferred orientation may exists in cellular division as well as competition between different cell populations. On the other hand, further spatial orchestration is needed in order to trans-

*e-mail: gerard.maugin@upmc.fr 
form random proliferations into a uniform growth, which must ultimately ceases as the correct size is reached. The control of shape can therefore depend both on cell number and on overall size, suggesting that the spatial gradient of morphogens can provide cells a dimension-sensing mechanism. This early vision was also supported by the discovery that cells have the ability to measure gradients comparing their own signalling level with those of their neighbours through specific regulatory pathways [8]. Nevertheless other interpretations are also possible. In fact, cells can change relative position undergoing a rearrangement process (e.g. during intercalation), implying that they are able to remodel their adhesive contacts making use of a mechanical feedback with their environment to adjust their position [9]. Giving a practical example, the stop signal for growth, thus determining the final size, could be triggered by a morphogen gradient level below a minimal threshold, as well as by a critical increase in tissue compression, causing a progressive inhibition of growth.

In summary, the orchestration of shape and size in the biological realm is more likely based on a combination of mechanical and biochemical feedbacks. Despite of the explosive rate of new knowledge on the biochemistry of morphogenesis, a major challenge is to understand the coordination between mechanical properties of the cells and the morphogenetic signals. In the following, we will discuss how the configuration forces theory can be used as a suitable theoretical framework for bridging this gap. The theoretical framework is defined in Sec. 2, and it is applied in Sec. 3 to a biological model system.

\section{Definition of the theoretical model}

The aim of this section is to introduce the proper kinematical description and the basic balance principles necessary to define a configurational-force theory of volumetric growth and mass transport inside a continuous body.

2.1. Kinematics. Let us consider a mapping $\mathbf{x}=f(\mathbf{X}, t)$ that provides the actual position $\mathbf{x}$ of a material point of a continuum body at time $t$, with position $\mathbf{X}$ in the reference configuration. The deformation field is described through the tensor gradient of deformation $\mathbf{F}=\operatorname{Grad} \mathbf{x}=\nabla_{X} \mathbf{x}$, and the second gradient of the deformation $\nabla_{X} \mathbf{F}=\nabla_{X} \nabla_{X} \mathbf{x}$. The mechanical problem for volumetric growth and mass transport can be formulated in material and spatial references, using two different space-time parametrizations: the so-called direct and inverse kinematics, respectively. The direct kinematics is based on the set of variables $(\mathbf{X}, t)$ belonging to the physical space, and the equations involves the spatial velocity $\mathbf{v}=\partial \mathbf{x} /\left.\partial t\right|_{X}$, and the material (spatial) velocity gradient $\mathbf{l}=\partial \mathbf{F} /\left.\partial t\right|_{X}\left(\mathbf{L}=\partial \mathbf{F} /\left.\partial t\right|_{X} \mathbf{F}^{-1}\right)$. On the other hand, the inverse kinematics is based on the inverse motion $\mathbf{X}=f^{-1}(\mathbf{x}, t)$, where the material domain changes over time keeping its range fixed. When dealing with inhomogeneities (or pseudo-inhomogeneities, in a more general framework) in the material setting, the theory of configurational forces demonstrates that the spatial parametrization is unable to ac- count for all the degrees of freedom associated to the deformation fields [10]. In particular, the physical linear momentum density $\mathbf{p}$, conjugated to the spatial velocity $\mathbf{v}$, is uniquely associated to the translational momentum. In the material manifold, the inverse motion velocity $\mathbf{V}$ can be defined through the identity:

$$
\begin{gathered}
\frac{d \mathbf{X}}{d t}=\frac{\partial f^{-1}(\mathbf{x}, t)}{\partial t}+\left.\frac{\partial f^{-1}(\mathbf{x}, t)}{\partial \mathbf{x}} \frac{\partial \mathbf{x}}{\partial t}\right|_{X}= \\
=\mathbf{V}+\mathbf{F}^{-1} \mathbf{v}=0 .
\end{gathered}
$$

When dealing with problems involving local rearrangements of the material manifold, we must therefore consider the balance of conjugated momentum $\mathbf{P}_{m}$, also referred as pseudomomentum density or canonical energy-momentum density. Furthermore, as accurately discussed in [11], a second gradient morphoelastic model is necessary if we want to include mass transport phenomena.

2.2. Balance principles. A continuum treatment of growth must account for a volumetric creation and/or absorption of mass (through source/sink terms in the balance equation) as well as for a surface flow, defined by a material flux M. The balance of mass in the material manifold is expressed in function of the time derivative (indicated as an upper dot) of the reference density $\rho_{0}$ at the material point in $\mathbf{X}$ :

$$
\dot{\rho}_{0}=\Gamma\left(c_{\alpha}, \nabla_{X} c_{\alpha}\right) \rho_{0}+\nabla_{\mathrm{X}} \cdot \mathbf{M}
$$

where $c_{\alpha}$, with $\alpha=1, \ldots . n$, are passive scalar fields determining the time- and space-dependent characteristics of growth inside the continuum. In our modeling framework, the scalars $c_{\alpha}(\mathbf{X}, t)$ can be seen as the concentration per unit of material volume of chemical substances (e.g. nutrients) and/or molecular signals (e.g. growth factors, morphogens) which are dispersed in the biological matter during the morphogenetic processes. In particular, the volumetric source of mass $\Gamma$ and the surface mass flux $\mathbf{M}$ may depend on both the local concentration and on the gradient of the internal variables, for consistency with the preliminary discussed experimental observations.

In terms of configurational forces, such scalar fields can be treated as internal variables in the expression of the free energy $\Psi$ per unit mass of the system, with $\Psi=$ $\Psi\left(\mathbf{F}, \nabla_{X} \mathbf{F}, c_{\alpha}, \nabla_{X} c_{\alpha}, \Theta ; \mathbf{X}, t\right)$. Here an explicit dependence both on the absolute temperature $\Theta$ and on $\mathbf{X}$ are considered (so that the material can be smoothly material inhomogeneous), while we drop an explicit dependence on time, discarding phenomena like ageing.

Indicating with $\mathbf{T}_{f}, \mathbf{T}_{s}$ the first Piola-Kirchhoff stress and hyperstress, i.e. the energy conjugates of $\mathbf{F}$ and $\nabla_{X} \mathbf{F}$, we can write the balance of mechanical energy of the body. Exploiting the principle of virtual powers and taking into account Eq. (2), we can write the local form for the balance of linear momentum in direct kinematics, as follows:

$$
\begin{gathered}
\frac{d}{d t}\left(\rho_{0} \mathbf{v}\right)=\frac{d \mathbf{p}}{d t}=\mathbf{f}_{0}+\Gamma \rho_{0} \mathbf{v} \\
+\nabla_{\mathrm{X}} \cdot\left(\mathbf{T}_{f}-\nabla_{\mathrm{X}} \cdot \mathbf{T}_{s}+\mathbf{M} \otimes \mathbf{v}\right),
\end{gathered}
$$




\section{Configurational forces for growth and shape regulations in morphogenesis}

where $\mathbf{f}_{0}$ represents the body force, and $\otimes$ is the dyadic product. In Eq. (3), we assumed that the internal variables have negligible inertia, so that they do not represent internal degrees of freedom (see [10], Ch. 5, for a broader discussion). As already discussed, a more suitable description of the evolution of material inhomogeneities requires a balance principle for the pseudo-momentum vector $\mathbf{P}_{m}=\rho_{0} \mathbf{F}^{T} . \mathbf{F}$.V. This balance equation can be obtained by a canonical projection of Eq. (3) in the material setting, and reads:

$$
\frac{d \mathbf{P}_{m}}{d t}=\mathbf{f}^{e x t}+\mathbf{f}^{g}+\mathbf{f}^{i n h}+\mathbf{f}^{c}+\mathbf{f}^{\Theta}+\nabla_{\mathbf{X}} \cdot \mathbf{b}
$$

Equation (4) states that there are five sources of material inhomogeneities: the convection of the body forces in $\mathbf{f}^{\text {ext }}$, the volumetric growth in $\mathbf{f}^{g}$, the true material inhomogeneities in $\mathbf{f}^{i n h}$, the internal variables in $\mathbf{f}^{c}$, and the temperature in $\mathbf{f}^{\Theta}$. They are defined as:

$$
\begin{gathered}
\mathbf{f}^{e x t}=-\mathbf{f}_{0} . \mathbf{F} \\
\mathbf{f}^{g}=\frac{\dot{\rho_{0}}}{\rho_{0}} \mathbf{P}_{m}-\left(\nabla_{X} \mathbf{v} \cdot \mathbf{M}\right) . \mathbf{F}, \\
\mathbf{f}^{i n h}=\left.\frac{\partial\left(K-\rho_{0} \Psi\right)}{\partial \mathbf{X}}\right|_{\text {expl }}, \\
\mathbf{f}^{c}=-\rho_{0}\left(\frac{\partial \Psi}{\partial c_{\alpha}} \nabla_{X} c_{\alpha}+\frac{\partial \Psi}{\partial\left(\nabla_{X} c_{\alpha}\right)} \nabla_{X} \nabla_{X} c_{\alpha}\right), \\
\mathbf{f}^{\Theta}=-\rho_{0} \frac{\partial \Psi}{\partial \Theta} \nabla_{X} \Theta=\rho_{0} \eta \nabla_{X} \Theta,
\end{gathered}
$$

where $K=1 / 2 \rho_{0} \mathbf{V} . \mathbf{C} . \mathbf{V}$ is the kinetic energy density, and $\eta$ is the entropy density per unit mass. The explicit derivative on $\mathbf{X}$ for $\mathbf{f}^{i n h}$ is defined keeping any other field constant. According to Eq. (4), the evolution of material inhomogeneities is driven by the second-gradient Eshelby stress tensor, defined as $\mathbf{b}=\mathbf{b}_{f}+\nabla_{\mathrm{X}} \cdot \mathbf{b}_{s}$, whose components read:

$$
\begin{gathered}
\mathbf{b}_{f}=-\left(K-\rho_{0} \Psi\right) \mathbf{I}_{R}-\mathbf{T}_{f} \cdot \mathbf{F}-2 \mathbf{T}_{s}:\left(\nabla_{X} \mathbf{F}\right) \\
\mathbf{b}_{s}=\nabla_{\mathrm{X}} \cdot\left(\mathbf{T}_{s} \cdot \mathbf{F}\right)
\end{gathered}
$$

In addition, we must impose that the Helmholtz free energy be frame-indifferent for arbitrary rotations of the actual configuration. This condition gives:

$$
\mathbf{F} . \mathbf{T}_{f}+\nabla_{X} \mathbf{F}: \mathbf{T}_{s}=\left(\mathbf{F} \cdot \mathbf{T}_{f}+\nabla_{X} \mathbf{F}: \mathbf{T}_{s}\right)^{T}
$$

and corresponds to a second-order balance principle for the angular momentum, imposing the symmetry of the generalized first-order Cauchy stress.

Let us now investigate the thermodynamic requirements for our growing continuum. Indicating with $\varepsilon$ the internal energy per unit mass, the local form of the first law of thermodynamics can be expressed as follows:

$$
\rho_{0} \dot{\varepsilon}=\mathbf{T}_{f}: \dot{\mathbf{F}}+\mathbf{T}_{s} \vdots \nabla_{X} \dot{\mathbf{F}}-\nabla_{\mathrm{X}} \cdot \mathbf{Q}+r_{0}+\mathbf{M} \cdot \nabla_{X} \varepsilon
$$

where $r_{0}$ is the external heat supply per unit of volume, and $\mathbf{Q}$ is the heat flux. Equation (9) is complemented by the local form of the second law of thermodynamics, imposing the following entropy inequality:

$$
\rho_{0} \dot{\eta} \geq \mathbf{M} \cdot \nabla_{X} \eta+\frac{r_{0}}{\Theta}-\nabla_{\mathbf{X}} \cdot\left(\frac{\mathbf{Q}}{\Theta}+\overline{\mathbf{Q}}\right),
$$

where $\overline{\mathbf{Q}}$ represents an 'extra' entropy flux, possibly driven by the diffusion of the internal variables inside the material. Recalling that the Helmholtz free energy per unit of mass is defined as $\Psi=\varepsilon-\Theta \eta$, we put together Eqs. (9), (10) obtaining the Clausius-Duhem form of the dissipation inequality for a second gradient continuum:

$$
\begin{gathered}
-\rho_{0}(\dot{\Psi}+\dot{\Theta} \eta)+\mathbf{T}_{f}: \dot{\mathbf{F}}+\mathbf{T}_{s} \vdots \nabla_{X} \dot{\mathbf{F}} \\
+\mathbf{M} \cdot\left(\nabla_{X} \Psi+\eta \nabla_{X} \Theta\right)-\frac{\mathbf{Q}}{\Theta} \cdot \nabla_{X} \Theta+\Theta \nabla_{X} \cdot \overline{\mathbf{Q}} \geq 0
\end{gathered}
$$

Equation (11) describes the thermodynamical restriction for the energy dissipation rate inside a growing second gradient hyperelastic continuum.

2.3. Constitutive equations with growth. As discussed in a preceding paper [12], volumetric growth can be modeled using a material isomorphism based on both a firstand a second-order transplant $\mathbf{F}_{g}$ and $\mathbf{Q}_{g}$, respectively. In this framework, the strain energy density is given by $\Psi=$ $\left(\operatorname{det} \mathbf{F}_{g}\right) \cdot \Psi_{0}\left(\mathbf{F}_{e}, \mathbf{Q}_{e}, c_{\alpha}, \nabla_{X} c_{\alpha}, \Theta\right)$, where the elastic terms $\mathbf{F}_{e}, \mathbf{Q}_{e}$ read as follows:

$$
\begin{gathered}
\mathbf{F}_{e}=\mathbf{F} \cdot \mathbf{F}_{g}^{-1} \\
\mathbf{Q}_{e}=\nabla_{R} \mathbf{F}:\left[\mathbf{F}_{g}^{-1}, \mathbf{F}_{g}^{-1}\right]-\mathbf{F}_{e} \cdot \mathbf{Q}_{g}:\left[\mathbf{F}_{g}^{-1}, \mathbf{F}_{g}^{-1}\right]
\end{gathered}
$$

from the composition laws of the first and second derivatives, where $(\mathbb{C}:[A, B])_{i j k}=\mathbb{C}_{i \alpha \beta} A_{\alpha j} B_{\beta k}$. In the geometrical line description, in Eq. (12) we introduce a first-order (generally not symmetric) material connection $\Gamma$, and a symmetric second-order linear connection $\boldsymbol{\Lambda}$, defined as:

$$
\boldsymbol{\Gamma}=-\mathbf{F}_{g}^{-1} \cdot \nabla_{X} \mathbf{F}_{g} ; \quad \boldsymbol{\Lambda}=\mathbf{F}_{g}^{-1} \cdot \mathbf{Q}_{g}
$$

representing the torsion and the curvature of the true material inhomogeneities. Using the transformation rules of Eq.(12) in the dissipation inequality, we derive the following constitutive equations in isothermal conditions:

$$
\begin{gathered}
\left(\mathbf{T}_{s}^{T}:\left[\mathbf{F}_{g}^{T}, \mathbf{F}_{g}^{T}\right]\right)^{T}=J \rho_{0} \frac{\partial \Psi_{0}}{\partial \mathbf{Q}}, \\
\mathbf{Q}_{g}: \mathbf{T}_{s}+\mathbf{F}_{g} \cdot \mathbf{T}_{f}=J \rho_{0} \frac{\partial \Psi_{0}}{\partial \mathbf{F}_{e}},
\end{gathered}
$$

while Eq. (11) can be simplified as:

$$
\begin{gathered}
-\widetilde{\mathbf{b}}_{f}:\left(\dot{\mathbf{F}}_{g} \mathbf{F}_{g}^{-1}\right)+\mathbf{M} \cdot \nabla_{X} \Psi+\mathbf{b}_{s} \vdots \dot{\boldsymbol{\Lambda}}+\Theta \nabla_{X} \cdot \overline{\mathbf{Q}} \\
-\rho_{0}\left(\frac{\partial \Psi}{\partial c_{\alpha}} \dot{c}_{\alpha}+\frac{\partial \Psi}{\partial\left(\nabla_{X} c_{\alpha}\right)} \nabla_{X} \dot{c}_{\alpha}\right) \geq 0 .
\end{gathered}
$$


In summary, the first-order Eshelby stress in the grown state, given by

$$
\widetilde{\mathbf{b}}_{f}=\rho_{0}\left(\psi \mathbf{I}-\frac{\partial \psi}{\partial \mathbf{F}_{e}} \cdot \mathbf{F}_{e}-2 \frac{\partial \psi}{\partial \mathbf{Q}_{e}}: \mathbf{Q}_{e}\right),
$$

and the material Eshelby hyperstress $\mathbf{b}_{s}$ drive the evolution of $\mathbf{F}_{g}$ and $\mathbf{Q}_{g}$, respectively. The Clausius-Duhem inequality in Eq. (15) provides the thermodynamic basis for understanding how the regulation mechanisms of growth and shape are coupled with both the mechanical and the biochemical state of the continuum. In the following, we discuss the role of configurational forces during morphogenesis in a simple model system.

\section{Application: the growth control in the imaginal wing disc of Drosophila}

The imaginal wing disc of Drosophila melanogaster is a flat pouch of tens of epithelial cells formed at the early larval stage of development. During the metamorphosis in the pupa, the cells inside the disc multiply their number by a factor one thousand in about four days, while the disc dimensions predetermine the final size of the adult fly. In this larval period, the disc growth is regulated by the morphogen Decapentaplegic (DPP), which is secreted in a central smooth boundary between two sets of cell compartments. While the local concentration of DPP is found to trigger growth by activating wing patterning genes, DDP also spreads in the disc, forming a concentration gradient which is somehow implicated in growth and shape regulations. The aim of this paragraph is to investigate such orchestration mechanisms using the proposed theoretical framework.

Experimental studies have observed that growth inside the imaginal disc is spatially homogeneous [13], so that we can set $\mathbf{F}_{g}=g(t) \mathbf{I}$, where $g(t)$ is a time-dependent scalar growth rate. Moreover, the relative position between cells is found to remain unaltered over time, allowing to impose $\mathbf{M}=0$ everywhere. For the sake of simplicity, we neglect the presence of second-order inhomogeneities in the growth process, setting $\mathbf{Q}_{g}=0$ and not evolving. The strain energy density per unit mass of the imaginal disc takes the form $\Psi=J \cdot \Psi_{0}\left(\mathbf{F}_{e}, c, \nabla_{X} c, \Theta\right)$, where $J=\left(\operatorname{det} \mathbf{F}_{g}\right)$ is the area increase of the disc and $c$ is the local material concentration of DDP. Using a suitable field-theoretic viewpoint we envisage an extra-entropy flux given by

$$
\overline{\mathbf{Q}}=\rho_{0} \frac{\partial \Psi}{\partial\left(\nabla_{x} c\right)} \dot{c} / \Theta
$$

so that the reduced dissipation inequality in Eq. (15) reads:

$$
-\widetilde{\mathbf{b}}_{f}:\left(\dot{\mathbf{F}}_{g} \mathbf{F}_{g}^{-1}\right)-\rho_{0} \frac{\delta \Psi}{\delta c} \dot{c} \geq 0,
$$

where the chemical potential associated to the morphogen, given by the functional derivative of $\Psi$, is coupled with the growth evolution. Guided by the experimental knowledge, reporting a morphogen gradient scaling with tissue size [14], we can postulate the following simplified form of the strain energy density:

$$
\begin{gathered}
\Psi_{0}\left(\mathbf{F}_{e}, c, \nabla_{X} c, \Theta\right) \\
=\frac{\tau}{2}\left(D \nabla_{X} c \cdot \nabla_{X} c+\gamma c^{2}\right)+\bar{\Psi}_{0}\left(\mathbf{F}_{e}, \Theta\right),
\end{gathered}
$$

where $\tau, D, \gamma$ are positive coefficients which may depend at most on the temperature. In agreement with Eq. (16), a dissipative evolution equation for the morphogen can be given in the material setting by:

$$
\dot{c}=-\frac{\delta \Psi}{\delta c} / \tau=-\gamma c+\nabla_{X} \cdot\left(D \nabla_{X} c\right)
$$

Eq. (18) is a mobility equation having both an intrinsic timescale $T=\gamma^{-1}$, e.g. the half-life of DDP inside the disc, and a typical lengthscale $L=\sqrt{D T}$, which may represent the decay length of the exponential decrease of DDP from the source in experiments. In the following, we will consider dimensionless equations using the variables $\bar{t}=t / T$ and $\overline{\mathbf{X}}=\mathbf{X} / L$. Wartlick et al. [14] have also reported that all cells in the disc measured the same temporal changes in DDP signalling, so we can give the solution of Eq. (18) using a variable separation $c(\overline{\mathbf{X}}, \bar{t})=c_{X}(\overline{\mathbf{X}}) \cdot c_{t}(\bar{t})$, which reads:

$$
\frac{\dot{c}_{t}(\bar{t})}{c_{t}(\bar{t})}=-1+\frac{\nabla^{2} c_{X}(\overline{\mathbf{X}})}{c_{X}(\overline{\mathbf{X}})}=\alpha,
$$

where $\alpha$ is a positive constant, which represents the space invariant temporal increase in DDP. Such a value is empirically found to correlate with the growth rate of cells, so that $\alpha \ll 1$, representing the ratio between the half-life time of DDP (about 30 minutes) and the doubling-time of cells inside the imaginal disc (about 4 hours) [14]. The exponential distribution $c_{X}$ is therefore completely defined imposing the growth-triggering value $C_{0}$ at the external border of the disc, together with the border continuity of $c$ and $\nabla c$ with the outer solution, which must vanish at long distances. This simple derivation is left as an exercise to the readers.

In absence of external geometrical constraints, the growth process does not generate residual stresses inside the imaginal disc, so that $\mathbf{F}_{e}=\mathbf{I}$ and $\widetilde{\mathbf{b}}_{f}=J \rho_{0} \Psi_{0} \mathbf{I}$. Moreover, we can postulate that $\rho_{0} \tau \dot{c}^{2}=\xi J \rho_{0} \Psi_{0}+\Sigma$, where $\xi$ represents the constant production rate of strain energy density per unit volume, so that $\Sigma \geq 0$ is the energy dissipation rate of the growth process. Considering that the cells keep their spatial density $\rho_{c}$ unchanged during the growth process, and recalling that $\rho_{0}=\rho_{c} \cdot J$, we derive from Eq. (16) the following constitutive equation for growth:

$$
\frac{\dot{J}}{J}=\frac{\dot{\rho}_{0}}{\rho_{0}}=\mathbf{I}:\left(\dot{\mathbf{F}}_{g} \mathbf{F}_{g}^{-1}\right)=\xi
$$

Putting together Eqs. (19), (20), we find that

$$
\log \left(\mathrm{c}_{\mathrm{t}}(\overline{\mathrm{t}})\right)=\frac{\alpha}{\xi} \log (\mathrm{J}(\overline{\mathrm{t}}))=2 \frac{\alpha}{\xi} \log (\mathrm{g}(\overline{\mathrm{t}})),
$$

which corresponds to the experimental curves in [15]. In particular, the cellular proliferation rate inside the disc is correlated to the temporal signalling increase in DDP through a system constant, experimentally measured at $\alpha / \xi \sim 0.59$.

In conclusion, we have demonstrated that the DDP morphogen controls growth and shape regulations inside the imaginal disc at the larval stage. An additional hypothesis can be 


\section{Configurational forces for growth and shape regulations in morphogenesis}

done on the control of its final size, which can be determined by the spatial gradient of $c$, given by $\nabla_{x} c=\mathbf{F}^{-T} \cdot \nabla_{X} c$. When $\nabla_{x} c$ decreases under a minimal threshold at the border, growth stops in an outer ring of the disc. Consequently, inner cells proliferation provokes residual compression inside the disc, which in turn gradually inhibits growth thanks to the Eshelbian coupling in Eq. (16). Further developments in this sense will make the object of a future work.

\section{Conclusions}

Developmental biologists made enormous progress over the past decades in deciphering the molecular bases of pattern formation; however, shape and size regulations are still poorly understood. In this work, we proposed a continuum model for growth and mass transport during morphogenetic processes. Using the theory of configurational forces, we have determined the thermo-mechanical bases of growth regulation mechanisms based on both the mechanical and the biochemical state of the tissue. The model is successfully applied to describe how the DDP morphogen in Drosophila melanogaster not only triggers growth of the imaginal wing disc, but can also control its shape and final size.

\section{REFERENCES}

[1] D'Arcy W. Thompson, On Growth and form, Cambridge Univ. Press, Cambridge, 1917.

[2] C.H. Waddington, "The distribution of the evocator in the unfertilized egg”, J. Exp. Biol. 15, 382-384 (1938).

[3] A.M. Turing, "The chemical basis of morphogenesis", Proc. R. Soc. Lond. Biol. Sci. B 237, 37-72 (1952).
[4] L. Wolpert, "Positional information and the spatial pattern of cellular differentiation", J. Theor. Biol. 25, 1-47 (1969).

[5] L. Wolpert, "Positional information and patterning revisited", J. Theor. Biol. 269, 359-365 (2011).

[6] S.J. Day and P.A. Lawrence, "Measuring dimensions: the regulation of size and shape", Development 127, 2977-2987 (2000).

[7] T. Lecuit and L. Le Goff, "Orchestrating size and shape during morphogenesis", Nature 450, 189-192 (2007).

[8] A.J. Zhu and M.P. Scott, "Incredible journey: how do developmental signals travel through tissue?", Gene Dev. 18, 19851992 (2004).

[9] T. Lecuit and P.F. Lenne, "Cell surface mechanics and the control of cell shape, tissue patterns and morphogenesis", Nature Rev. Mol. Cell. Biol. 8, 633-644 (2007).

[10] G.A. Maugin, Configurational Forces: Thermomechanics, Physics, Mathemathics and Numerics, CRC Press Taylor and Francis, Boca Raton, 2010.

[11] P. Ciarletta and G.A. Maugin, "Elements of a finite strain gradient thermomechanical theory for material growth and remodelling", Int. J. Nonlinear Mech. 46, 1341-1346 (2011).

[12] P. Ciarletta, D. Ambrosi, and G.A. Maugin, "Mass transport in morphogenetic processes: a second gradient theory for volumetric growth and material remodelling", J. Mech. Phys. Solids 60 (3), 432-450 (2012).

[13] L. Le Goff and T. Lecuit, "Gradient scaling and growth", Science 331, 1141-1142 (2011).

[14] O. Wartlick, P. Mumcu, F. Julicher, and M. Gonzalez-Gaitan, "Understanding morphogenetic growth control - lessons from flies”, Nature Rev. Mol. Cell. Biol. 12, 594-604 (2011).

[15] O. Wartlick, P. Mumcu, A. Kicheva, T. Bittig, C. Seum, F. Julicher, and M. Gonzalez-Gaitan, "Dynamics of Ddp signalling and proliferation control", Science 331, 1154-1159 (2011). 